\title{
A Method of Multidimensional Knowledge Push for Product Designers
}

\author{
Yuzhong Jiang ${ }^{1, \text { a }}$, Yan Yan $^{1}$, Yu Dong ${ }^{2}$, Guoxin Wang ${ }^{1}$, Jia Hao ${ }^{1}$ \\ ${ }^{1}$ School of Mechanical Engineering, Beijing Institute of Technology, Beijing 100081, China \\ ${ }^{2}$ China North Vehicle Research Institute, Beijing 100072, China \\ aiyuzhong@foxmail.com
}

Keywords: knowledge management; knowledge push; forgotten attribute; knowledge engineering; similar filter.

\begin{abstract}
A knowledge push framework based on analysis of the behavior, task and role of a designer was proposed, providing excellent ways for solving the problems of low accuracy, complicated operation, low efficiency and difficulty in acquiring knowledge through retrieval. A knowledge active push system was realized through forgotten attenuation, similarity filtering and other key methods, playing a good supporting role to designers and greatly reducing the time required for designing.
\end{abstract}

\section{Introduction}

With increasing popularization and application of information technology in depth, more and more knowledge-intensive enterprises and research institutions begin to pay much more attention to knowledge management. In the field of design, designers often need to use a lot of knowledge in order to make a satisfactory design. However, with the accumulation of time and a growing number of knowledge, designers have been facing a huge number of knowledge. The inability to effectively get the required knowledge is an increasingly apparent contradiction. The current search has been unable to meet the needs of designers ${ }^{[1]}$. It has become a hot spot and trend to set up knowledge push system to meet the user knowledge requirement in knowledge management.

Knowledge push does not pull off in the traditional way. Through knowledge push knowledge migrating can be accelerated, phenomenon of knowledge asymmetry eliminated and "knowledge excess" overcome, which effectively meet the knowledge demand of design staff and implement knowledge transfer and sharing and creation. Many scholars made a lot of research on how to push knowledge to design staff for utilizing in product design process. Wang Shengfa proposed a knowledge pushing method based on workflow ${ }^{[1]}$. Feng Yong Proposed a knowledge push system based on ontology and knowledge grid ${ }^{[2,3]}$. Pan Min created a variant design knowledge pushing method ${ }^{[4]}$. Brandt, Sebastian C put forward a kind of knowledge reuse mechanism based on Ontology ${ }^{[5]}$, and Zhang Dongmin provides a knowledge transfer and reuse prototype system. However, these methods and systems used in the design companies failed to cover dimensions of knowledge needs of design personnel; and the establishment of user model accumulated with time tend to be too large, so new interest is hard to feedback to the user model, leading to knowledge push accuracy dropping. In addition, a list of pushed knowledge contains very much approximate knowledge, making the diversity of content seriously insufficient.

\section{Multiple dimensions of knowledge push frame}

Analysis of knowledge push is running mainly from design personnel's behavior, tasks with context and roles three dimensions. According to these dimensions, a workflow driven push architecture allowing task decomposition is proposed based on knowledge management as shown in Figure 1, whose whole structure from up to down contains the workflow layer, user layer, push the computation layer, knowledge management layer and the knowledge layer. 


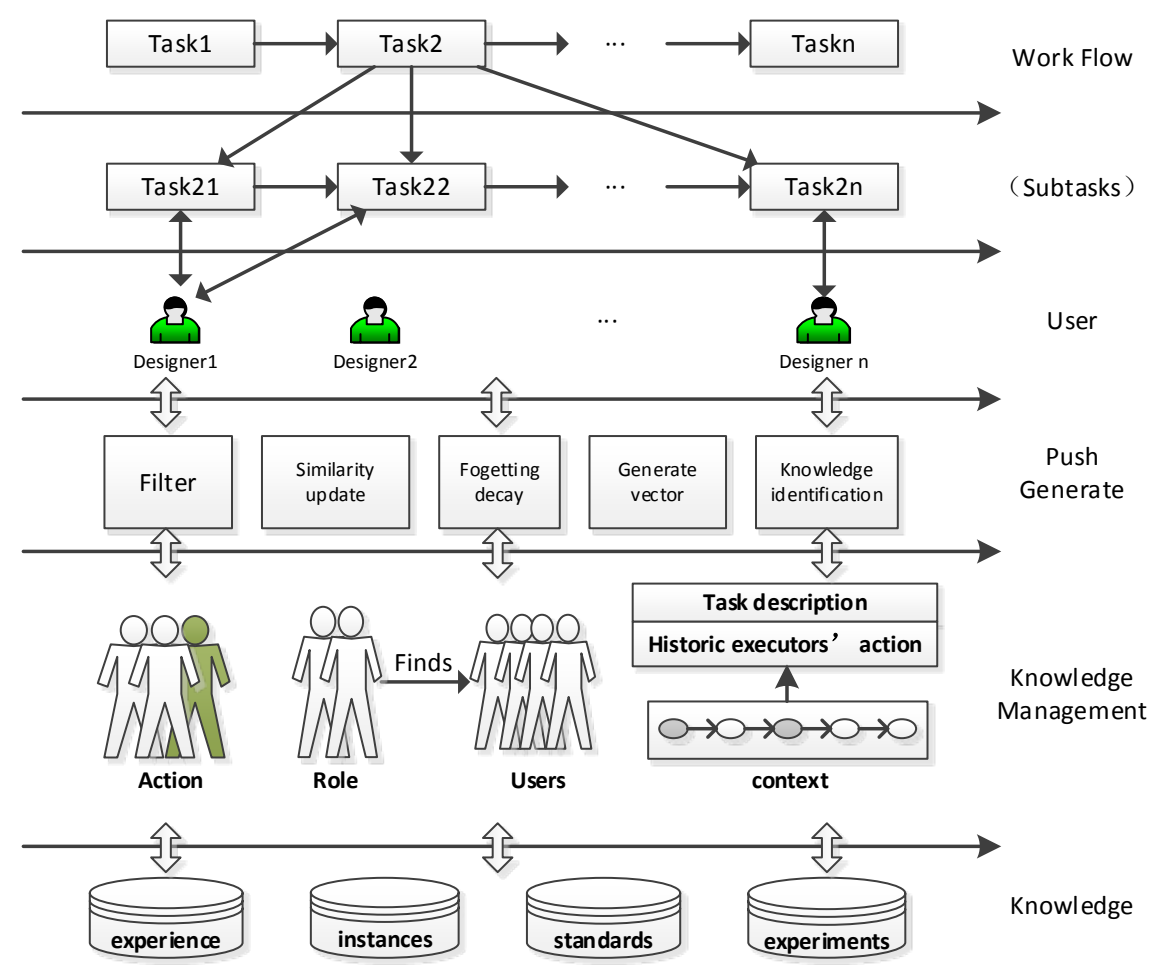

Figure 1 A multi-dimensional framework for designers

Collecting user behavior. It is a complex process to collect user behavior. Firstly there need to be construction of user behavior in the knowledge management system including collection, comment, sharing, and focus; Secondly, get users' historical behavior from the user behavior database and knowledge vector generated from the corresponding knowledge, superpose them according to the time order and accomplish the formation of user behavior vector. During every superposition, if a vector is a keyword already exists, it will run forgotten attenuation.

Collecting task and contextual information. As already mentioned above, the knowledge push content not only related with the designers, but also their currently executing tasks. Collecting tasks information is similar to that of users, and information of tasks' historical executors' behavior needs to be gathered. After collecting this information and the fusion with task description vector, eventually tasks' vector model is formed. Considering characteristics of tasks with the context, unlike the collecting user behavior, the information of upstream and downstream tasks will also be analyzed at the same time.

User role mapping In addition to the collection of user behavior and task related information, user roles in the system are obtained automatically. Mapping based on roles and positions, knowledge for posts will be pushed to users.

\section{Key methods to push}

Vector model. A component coordinate of vector model of knowledge demand can be named after a keyword; vector length represents the weight of the keyword.

In this paper, the traditional vector model has a certain degree of modification, adding synonym, thesaurus and antonym judgment logic. For example, "Computer Aided Design" and "CAD" is synonymous, then the two coordinate names as the same coordinate (or in the same direction), whose inner product is the product of multiplying respective length.

For example, "knowledge push" and "knowledge management" are synonyms. Although the coordinates named respectively after them are different, he result is multiplied by the direction vectors is not zero but a set value. If we set "fast" and "slow" as antonyms, the product of these two coordinates is the opposite number of the product of multiplying respective length (they are considered on the same line, but in the opposite direction). 
Amnestic vector model of the user model As vector model need to be accurate description for users' states, considering that a user as a natural person having the idiosyncrasy of oblivion, vector model should also be given that peculiarity.

Shan Rong also presented a forgotten user model ${ }^{[6]}$. But its formulae have many parameters to be set. This paper put forward a whole new formula for vector model. Lessoning from the famous Ebbinghaus forgetting curve, simplified it to some extent (properly screen out the meaningless memory according to the forgetting theory that is complicated and whose attributes are difficult to distinguish, and retain the meaningful memory).

Vector model $\mathrm{V}=\{\mathrm{c} 1, \mathrm{c} 2, \ldots, \mathrm{cn}\}$, where each ci for different keywords in the component.

Each vector component ci consists of four units $\{\mathrm{Wdi}, \mathrm{Wti}, \mathrm{Fi}, \mathrm{Ti}\}$, where Wdi is the keyword (word), Wti is the weight of the word at the moment of Ti (weight), Fi is the word frequency (Frequency), and Ti is the word's last update time (Time). The module of component ci, that is the weight of Wdi, is a variable updates in real-time, continuing to decay over time.

The ci module is defined as:

$$
\left|c_{i}\right|=\frac{W t_{i}}{\left(T_{\text {now }}-T_{i}\right)^{1 / \lg \left(F_{i}+1\right)}}
$$

Where Ti is the time of the last update, Tnow is the time now, the unit of (Tnow - Ti) is day, and its value is regarded as 1 day if less than 1 day (the effect is only after 1 days the weight decay start).

Vector module, namely its real-time weight, based on the principle of Occam's razor, uses the relatively simple reciprocal relationship, but meets two main rules for oblivion:

Attenuation velocity slow down over time;

With the increase in the number of repeat (review), attenuation curve becomes more flat.

Similar filter of pushed knowledge. For a set of knowledge whose similarity is higher than threshold, only one piece of the most representative knowledge will be pushed out. Among them, there are two kinds of definitions of representative:

(1) The similarity between vector of this piece of knowledge and others' vectors' summation within the group;

(2) The similarity between this piece of knowledge and user model.

Evaluation of knowledge push. This paper recommended mandatory evaluation (user has the right to shut the mandatory evaluation down). Hidden behavior of users is often elusive, but user's explicit behavior is easier to demonstrate the degree of users' satisfaction. The chance false positives will reduce when appraisal data become the basis of feedback of iteration. When user does not evaluate or refuse to carry out evaluation, viewing time of user's browsing or other operations is used to judge the quality of push. So, regarding push feedback from user, implicit acts are used to expand the scope of content and explicit acts are used to significantly improve the accuracy of feedback. These two mutually assist, to make up for each other's disadvantages.

\section{Case Study}

On the Distributed Intelligence Resource-driven Innovative Design Platform (DIRIDP) at Beijing $\mathrm{XX}$ Institute, according to this paper, based on the analysis of knowledge push demands and knowledge classification, both a traditional push system without the key methods above and one with the methods in this paper were implemented. The implementation guaranteed as far as possible that the ability of personnel, task difficulty, workload and referable knowledge for each group are balanced.

Through collection and analysis of relevant data before and after the application of the push system, implementation of push methods in this paper, compared with before implementation and the implementation of traditional knowledge push to designers, the average time to complete tasks sharply declined, the amount of knowledge referenced reduced, obviously improved the coverage of knowledge, significantly improved the efficiency in obtaining to knowledge, and effectively supported the use of knowledge. 


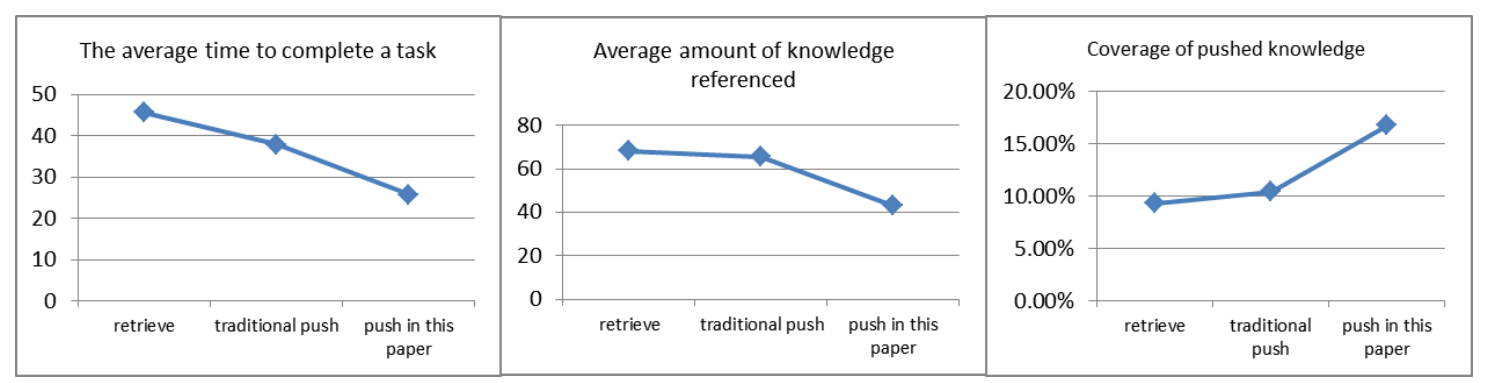

Figure 2 multi-dimensional knowledge push approaches effect contrast

\section{Conclusion}

A knowledge push system is proposed based on user behavior, tasks and roles these three dimensions and some additional key attributes. Through amnestic vector model that contains extension words, complete updating mechanism and similar filtering matching mechanism, the push diversity, coverage and accuracy are largely improved. Also, the efficiency and quality of designers' obtaining knowledge in the implementation of tasks is promoted and time to complete design tasks is shortened. The push method in this paper effectively assists designers to finished tasks, which is an effective way for knowledge intensive enterprises and research institutions to promote work efficiency. Further works include: to optimize the speed of knowledge push calculation, to integrate more methods which is suitable for knowledge pushing to designers, such as packaging knowledge and software programs as a whole unit to push, and to perfect the prototype method for mechanical design knowledge push, such as pushing knowledge on the 3D-model.

\section{Acknowledgments}

This research work is supported by projects (A2220133001) funded by National Ministries and Commissions and projects (51375049) funded by the National Science Foundation of China. The authors are grateful to the anonymous reviewers for their kind comments on this paper.

\section{References}

[1] Sheng-Fa Wang, Xin-Jian G. U.,Jian-Feng Guo,etc. Knowledge active push for product design [J]. 2007, 13(2): 234-239. (In Chinese)

[2] Yong Feng,Zhi-Ping Fan,Bo Feng,etc. Construction of knowledge push system in customer service center of enterprise [J]. 2007, 13(5): 1015-1020. (In Chinese)

[3] Yong Feng, Hong-Yan X. U.,Miao Zhao. An Architecture Platform of Knowledge Push System for Knowledge-based Organization's Post [J]. 2006, 33(3): 269-272. (In Chinese)

[4] Min Pan,Sheng-Fa Wang, Fan-Guo Kong. Research on active propelling of knowledge for variant design [J]. 2011(8): 119-121.(In Chinese)

[5] Brandt S C, Morbach J, Miatidis M, et al. An ontology-based approach to knowledge management in design processes [J]. 2008, 32(1-2): 320-342.

[6] SHAN Rong. Research of user interest model update and forgetting mechanism [J]. 2011(07): 10-11.(In Chinese) 